\title{
Threat assessment for avoiding collisions with turning vehicles
}

\author{
Mattias Brännström, Erik Coelingh and Jonas Sjöberg
}

\begin{abstract}
This paper presents a method for estimating how the driver of a vehicle can use steering, braking or acceleration to avoid a collision with a moving object. In the method, the motion of the object can be described with an arbitrary motion model and polygons are used to describe its expected extension. The key idea is to parameterize the motion of the vehicle such that an analytical solution can be derived for estimating the set of manoeuvres that the driver can use to avoid the object at discrete times. The union of the solutions for all times is used to estimate how a collision can be avoided during the complete prediction horizon. Additionally, a decision-making algorithm is proposed that decides when to initiate autonomous braking to avoid or mitigate a potential collision. A collision avoidance by braking system, based on the proposed method and algorithm, has been evaluated on simulated traffic scenarios at intersections. It is shown that a vehicle equipped with such a system can potentially reduce the impact velocity with up to $40 \mathrm{~km} / \mathrm{h}$ in left turn across path situations.
\end{abstract}

\section{INTRODUCTION}

The World Health Organization estimates that traffic accidents annually cause 1.2 million fatalities and as many as 50 million injuries. It is predicted that these figures will increase by $65 \%$ over the next 20 years unless there is new commitment to prevention [1]. Accident research has shown that some accidents are caused by vehicle failures, but most accidents are caused by human errors [2]. One way to reduce the number of accidents and their consequences is to actively assist road users in their driving task. This is called active safety or in more general terms preventive safety, ranging from electronic stability control to drowsiness detection systems.

An important subset of preventive safety is collision avoidance systems, aiming at assisting the driver in avoiding collisions with e.g. vehicles, bicyclists or pedestrians [3]. Several vehicle manufacturers now offer functionality that help the driver in avoiding collisions by providing warnings, brake support or even automatic intervention braking. These technologies are often applied to rear-end collision situations. One reason behind this is that rear-end collisions are common accident scenarios which are generally estimated to account for $23 \%$ of all police-reported accidents and $5 \%$ of all fatalities [4]. Another reason is that the kinematics of rear-end collisions are relatively easy to predict, making threat assessment and decision-making more straightforward. Threat assessment is commonly used denotation for algorithms that estimate how road users can act to avoid a potential collision.

State-of-the-art collision avoidance technology relies on invehicle sensors like radar and camera that constantly monitor

M. Brännström and E. Coelingh are with the Department of Vehicle Dynamics and Active Safety, Volvo Car Corporation, 40531 Göteborg, Sweden mbranns3@volvocars.com, ecoeling@volvocars.com

J. Sjöberg and M. Brännström are with the Department of Signals and Systems, Chalmers University of Technology, 41296 Göteborg, Sweden jonas.s joberg@chalmers.se the area in front of the car. If the car approaches another vehicle and the driver needs to undertake an action to avoid an accident, an audible and/or visible warning is provided in order to increase the driver's attention in such a way that he or she can avoid or mitigate the accident [5]. If the driver does not react to the warning and it is judged that the driver is unable to avoid a collision without assistance, autonomous braking is applied to mitigate or avoid the potential collision.

To further increase the scope of collision avoidance technology this paper focuses on threat assessment for avoiding or mitigating collisions at intersections and roundabouts.

Compared to rear-end collision the differences are:

- In rear-end collisions only the rear-side of the lead vehicle is of interest and this side is always approached at a 90 degrees angle. The orientation of a vehicle entering an intersection may change over time and thus, the front, rear and side of the vehicle has to be taken into account.

- The driver of the lead vehicle has limited possibilities to detect the striking vehicle. The driver of a crossing vehicle has better possibilities to detect potential threats.

Collisions at intersections account for $41 \%$ of all policereported crashes, $46 \%$ of all injuries and $21 \%$ of all fatalities $[6,7]$. Several algorithms for assessing traffic situations at intersections has been proposed in previous research. Some threat assessment algorithms are restricted to straight crossing path scenarios, where deterministic constant acceleration models are used to describe the motion of the crossing vehicle [8]. The simplified motion model makes is possible to derive an analytical solution. Algorithms that can assess more general traffic scenarios often use a brute fore approach, e.g. MonteCarlo simulations [9], to find potential avoiding manoeuvres. The analytical algorithms can easily be implemented in realtime applications, while the brute force algorithms are much more computationally demanding.

\section{OUtLine}

The key idea of the method presented in this paper is to discretize a multi-dimensional threat assessment problem into several smaller problems which can be solved analytically. Using analytical solutions makes the method a computationally efficient alternative for assessing complex traffic scenarios, e.g. involving turning vehicles at intersections or roundabouts.

In Section III, a decision-making algorithm is proposed determining when to initiate autonomous braking. Decisionmaking algorithms for autonomous steering or acceleration are not considered in this paper. Section IV contains the proposed method for estimating if the driver of a vehicle can use steering, braking or acceleration to avoid a collision with a moving object. In Section $\mathrm{V}$, a collision avoidance by braking system, based on the proposed method and algorithm, 
is evaluated on simulated traffic scenarios. Finally, conclusions are drawn in Section VI.

\section{DECISION MAKING FOR AUTONOMOUS BRAKING}

Assume that the driver of a vehicle needs to avoid a collision with an object during a limited prediction horizon, $t \in\left[0, T_{\max }\right]$. Denote the vehicle as the host vehicle, the object as a target and assume that there are no other obstacles present. Assume that the driver of the host vehicle or the driver of the target vehicle can attempt to avoid a collision by either braking, steering or acceleration. Potential coordinated avoidance manoeuvres, where both the host vehicle and the target vehicle performs synchronized avoiding manoeuvres are not assessed. Nor are potential combined braking and steering manoeuvres. Although these assumptions may appear too limiting to be useful in practice, they are quite reasonable when assessing critical traffic situations. If the prediction horizon is sufficiently small, it is reasonable to neglect the possibility to initiate coordinated avoidance manoeuvres.

Instead of considering coordinated avoidance manoeuvres, it is proposed that the traffic situation shall be assessed both from the host vehicle perspective and the target perspective. First, the set of host vehicle manoeuvres that can be used to avoid a collision is estimated, under the assumption that the future trajectory of the target is given by the current state of the target. Secondly, the set of target manoeuvres that can be used to avoid a collision is estimated, under the assumption that the future trajectory of the host vehicle is deterministic and given by the current state of the host vehicle.

Like earlier developed methods [3], interventions are inhibited if the driver of the host vehicle has the opportunity to avoid a collision. The decision-making algorithm presented in this paper also inhibits interventions if the target has the opportunity to avoid a collision. This is a conservative approach which reduces the risk of unnecessary interventions, especially at intersections where there is a possibility that the driver of a crossing vehicle detects the threat and makes a late avoiding manoeuvre.

In general, it is easier for either of the driver of the host vehicle or the driver of the target vehicle to perform a late avoiding manoeuvre. For example, assume that the host vehicle is approaching a stationary target in a rear-end collision situation. Then, it is always easier for the driver of the host vehicle to perform an avoiding manoeuvre than the other way around.

Autonomous braking is proposed to be initiated when:

- The driver of the target vehicle can not avoid a collision by steering, braking or accelerating, AND

- The driver of the host vehicle can not avoid a collision by steering or accelerating, AND

- Full braking is needed in the host vehicle to avoid a collision.

This algorithm assures that if both vehicles are equipped with the similar algorithms, potential interventions in the vehicles will not interfere with each other in e.g. crossing traffic situations. The autonomous braking is interrupted when the host vehicle no longer need to brake to avoid a collision.

\section{THREAT ASSESSMENT}

In this section, a method is proposed for estimating how the driver of a vehicle can use steering, braking or acceleration to avoid a collision during a limited prediction horizon. The method is used to derive a threat assessment algorithm. For simplicity, the algorithm is described the host vehicle perspective, but it can easily be modified to assess the situation from a target vehicle perspective.

In Section IV-A, the motion model for the host vehicle is described and needed assumptions are made. Section IV-B contains the method for estimating how the driver of the vehicle can use steering to avoid a collision. In Section IV-C, the method is extended to estimate how the driver can brake or accelerate to avoid a collision.

Assume that the host vehicle has access to good estimates of the target state, e.g. its dimensions, velocity, acceleration, yaw rate and orientation. These estimates can be obtained through a good in-vehicle sensor fusion system, possibly combined with vehicle to vehicle communication.

The proposed method has the following characteristics:

- Arbitrary motion models can be used for describing the motion of the target. This ensures that it potentially can be applied to rear-end collisions, collisions with turning objects, but also other collision scenarios.

- The target is represented by a polygon with arbitrary shape and number of vertices. These can represent different road users such as passenger vehicles, trucks, trucktrailer combinations as well as pedestrians and bicyclists.

- The polygonal shape of the target is allowed to change over time. This is an important characteristic when e.g. a turning truck-trailer combination is to be represented.

- The vehicle dynamics of the host vehicle is described by a so-called bicycle model [10], such that vehicle slip can be taken into account when judging the possibilities for collision avoidance by steering.

- The brake system dynamics of the host vehicle are taken into account to be able to realistically judge to possibilities for collision avoidance by braking.

\section{A. The vehicle model}

When using the proposed threat assessment method described in Section IV-B and IV-C, the motion of the host vehicle has to be parameterized such that only one solution exist for traveling to a certain location in a certain time. One parametrization is used for assessing the possibility to avoid a collision by steering and another parametrization is used for assessing collision avoidance by braking/acceleration. By using the selected parameterizations, the collision avoidance problem can be solved analytically. In this paper, a bicycle model has been selected to describe the motion of the host vehicle, as illustrated in Fig. 1.

Let the origin of a ground-fixed cartesian coordinate system $(x, y)$ be positioned at the initial position of the front center of 


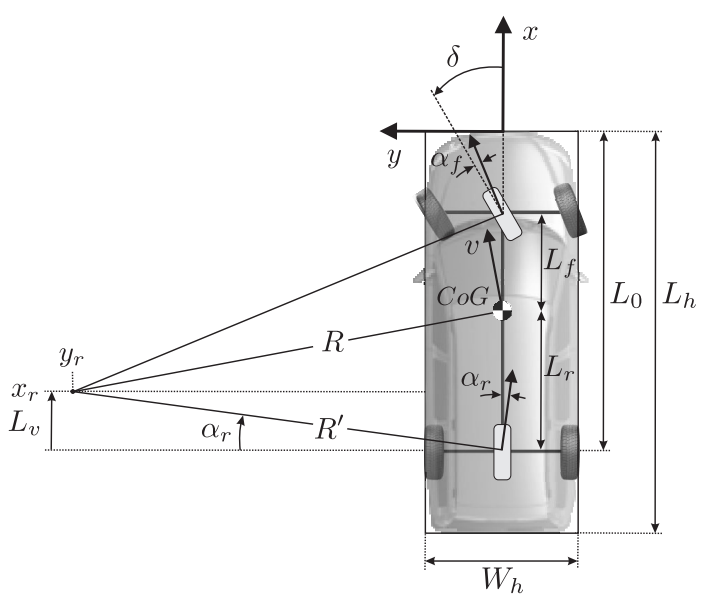

Fig. 1. The motion of the host vehicle is described with a bicycle model.

the host vehicle. The coordinate system is directed along the center of the initial orientation of the host vehicle.

The length and width of the host vehicle are indicated by $L_{h}$ and $W_{h}$. The center of gravity is denoted as $C o G$. The distance from the rear axle to $C o G$ is given by $L_{r}$. The distance from the front axle to $C o G$ is given by $L_{f}$. The distance from the front of the vehicle to the rear axle is $L_{0}$. The rear and front slip angles are given by $\alpha_{r}$ and $\alpha_{f}$, respectively. The steering wheel angle can be approximated with

$$
\delta=\alpha_{r}-\alpha_{f}+\frac{L_{f}+L_{r}}{R}
$$

where $R$ is the radius of the turn. The rear slip angle is given by

$$
\alpha_{r}=-\frac{M L_{f}}{\left(L_{f}+L_{r}\right) C_{r}} \frac{v^{2}}{R}
$$

where $M$ is the host vehicle mass and $C_{r}$ is the rear cornering stiffness. The front slip angle is given by

$$
\alpha_{f}=-\frac{M L_{r}}{\left(L_{f}+L_{r}\right) C_{f}} \frac{v^{2}}{R}
$$

where $C_{f}$ is the front cornering stiffness [10].

When turning, the dynamics is modeled with a time delay $t_{d}$ after which steady-state cornering is obtained instantaneously. Steady-state cornering, means that the turn center of the host vehicle, $\left(x_{r}, y_{r}\right)$, does not change over time and the steering angle $\delta$ is kept constant. The initial time delay can be used to compensate for the transient behavior until steady-state cornering is achieved, e.g. $t_{d}=0.3 \mathrm{~s}$. Time delays are easy to include in the algorithm and will be left out in the derivation to make the paper easier to follow.

The $x$-coordinate of the turn center is given by

$$
x_{r}=L_{v}-L_{0}
$$

where

$$
L_{v}=\left|R^{\prime} \sin \alpha_{r}\right| \approx\left|R \alpha_{r}\right|
$$

under the assumption that $\alpha_{r}$ is small and $R^{\prime} \approx R$ in Fig. 1 . Using (2) and (5) gives

$$
L_{v} \approx \frac{M L_{f}}{\left(L_{f}+L_{r}\right) C_{r}} v^{2}=k v^{2}
$$

where $k \geq 0$ is a constant that only depends on the vehicle weight distribution and the rear cornering stiffness.

The approximation (6) can be used for both oversteered and understeered vehicles during steady-state cornering. Note that the distance $L_{v}$ can exceed the length of the vehicle during normal driving conditions and that the approximation of $L_{v}$ is independent of the radius of turn $R$.

\section{B. Steer to avoid}

In this section, a method and an algorithm is proposed for estimating how the driver of the host vehicle can use steering to avoid a collision with a target vehicle.

The method consist of these steps:

1) Predict the motion of the target vehicle with an arbitrary motion model during a limited prediction horizon, $t \in$ $\left[0, T_{\max }\right]$.

2) Divide the prediction horizon into a series of discrete time steps $t_{i}=i t_{s}, e . g . t_{s}=0.05 s$ where $i \in 1,2, . ., N$ and $t_{s}=T_{\max } / N$, as illustrated in Fig. 2.

3) Let the target be represented by a polygon of arbitrary shape and number of vertices. The positions of the vertices at time $t_{i}$ are given by $\left(\mathbf{x}_{i}, \mathbf{y}_{i}\right)$. The shape of the polygon can be changed at each time step.

4) For each time step, $t_{i}$, find all vertices in $\left(\mathbf{x}_{i}, \mathbf{y}_{i}\right)$ that the host vehicle has to avoid and denote them as $\left(\mathbf{x}_{i}^{\prime}, \mathbf{y}_{i}^{\prime}\right)$.

5) For each time step, $t_{i}$, estimate how the driver of the host vehicle can steer to clear all selected vertices, $\left(\mathbf{x}_{i}^{\prime}, \mathbf{y}_{i}^{\prime}\right)$, either to the left or to the right. Both the front end and the rear end of the host vehicle shall clear all vertices, as illustrated in Fig. 3-4.

6) The union of the solutions in step 5 is used to estimate how the driver can steer to clear all selected vertices during the entire prediction horizon.

Step 1-3 are straightforward and needs no further explanation. Step 4: Only vertices that potentially could be reached at time $t_{i}$ has to be avoided. Assume that the host vehicle speed, $v$, is constant while the host vehicle is steering to avoid a collision. Thus, all vertices where $v t_{i}-L_{h}<\mathbf{x}_{i}<v t_{i}$ are reachable at time $t_{i}$. Denote such vertices as $\left(\mathbf{x}_{i}^{\prime}, \mathbf{y}_{i}^{\prime}\right)$.

Step 5: Potential steering manoeuvres are parameterized such that an analytical solution can be derived for estimating the manoeuvre needed for avoiding a single corner $\left(x_{i}, y_{i}\right) \in$ $\left(\mathbf{x}_{i}^{\prime}, \mathbf{y}_{i}^{\prime}\right)$. When using a bicycle model with steady-state cornering, the solution is given geometrically. A study of the Figures 3 and 4 gives that

To pass the corner $\left(x_{i}, y_{i}\right)$ on the left side with the front end of the host vehicle, the turn center is given by

$$
y_{r}^{\text {left, } \text { front }}=\frac{\left(x_{i}-x_{r}\right)^{2}+y_{i}^{2}-x_{r}^{2}-\frac{W_{h}^{2}}{4}}{2 y_{i}+W_{h}}
$$




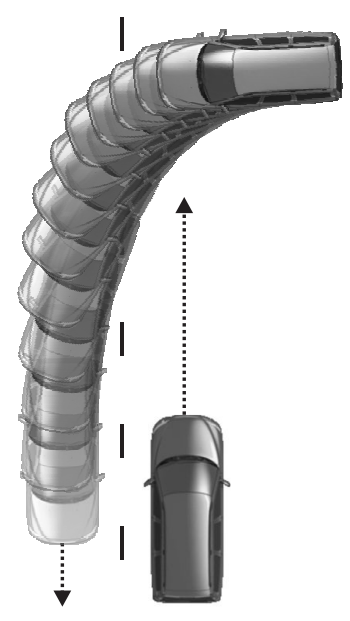

Fig. 2. Example: The host vehicle, illustrated at $t=0$, is approaching a turning target. The future position of the target is predicted in discrete time steps, $t_{i}$, during a limited prediction horizon $t \in\left[0, T_{\max }\right]$. The proposed method is used to find the set of steering, braking and acceleration manoeuvres that the host vehicle can use to avoid a collision during the entire prediction horizon.

To pass a corner $\left(x_{i}, y_{i}\right)$ on left hand side with the rear end of the host vehicle, the turn center is given by

$$
y_{r}^{\text {left, rear }}=\frac{\left(x_{i}-x_{r}\right)^{2}+y_{i}^{2}-\frac{W_{h}^{2}}{4}}{2 y_{i}+W_{h}}
$$

Similarly, to pass the corner on the right hand side, the turn center is given by

$$
y_{r}^{\text {right,front }}=\frac{\left(x_{i}-x_{r}\right)^{2}+y_{i}^{2}-x_{r}^{2}-\frac{W_{h}^{2}}{4}}{2 y_{i}-W_{h}}
$$

and

$$
y_{r}^{\text {right,rear }}=\frac{\left(x_{i}-x_{r}\right)^{2}+y_{i}^{2}-\frac{W_{h}^{2}}{4}}{2 y_{i}-W_{h}}
$$

Store the solutions for all time steps and all selected vertices in vectors $\mathbf{y}_{r}^{\text {left,front }}, \mathbf{y}_{r}^{\text {left,rear }}, \mathbf{y}_{r}^{\text {right,front }}$ and $\mathbf{y}_{r}^{\text {right,rear }}$.

Step 6: To pass all reachable vertices, $\left(\mathbf{x}_{i}^{\prime}, \mathbf{y}_{i}^{\prime}\right) \forall i \in 1,2, . ., N$, on left hand side, the turn center is given by

$$
y_{r, \text { left }}=\max \left(\left[\mathbf{y}_{r}^{\text {left,front }} \mathbf{y}_{r}^{\text {left,rear }}\right]^{-1}\right)^{-1}
$$

To pass on the right hand side, the turn center is given by

$$
y_{r, \text { right }}=\min \left(\left[\mathbf{y}_{r}^{\text {right,front }} \mathbf{y}_{r}^{\text {right,rear }}\right]^{-1}\right)^{-1}
$$

The avoiding manoeuvre is assessed as feasible if the corresponding lateral acceleration does not exceed the maximum allowed lateral acceleration, i.e. $a_{\mathrm{lat}} \leq a_{\mathrm{lat}}^{\max }$. The lateral acceleration to pass the target to the left is given by $a_{\text {lat }}^{\text {left }}=v^{2} / R_{\text {left }}$, and to the right $a_{\text {lat }}^{\text {right }}=v^{2} / R_{\text {right }}$, where

$$
R_{\text {left }}=\operatorname{sign}\left(y_{r, \text { left }}\right) \sqrt{y_{r, \text { left }}^{2}+\left(L_{r}-L_{v}\right)^{2}}
$$

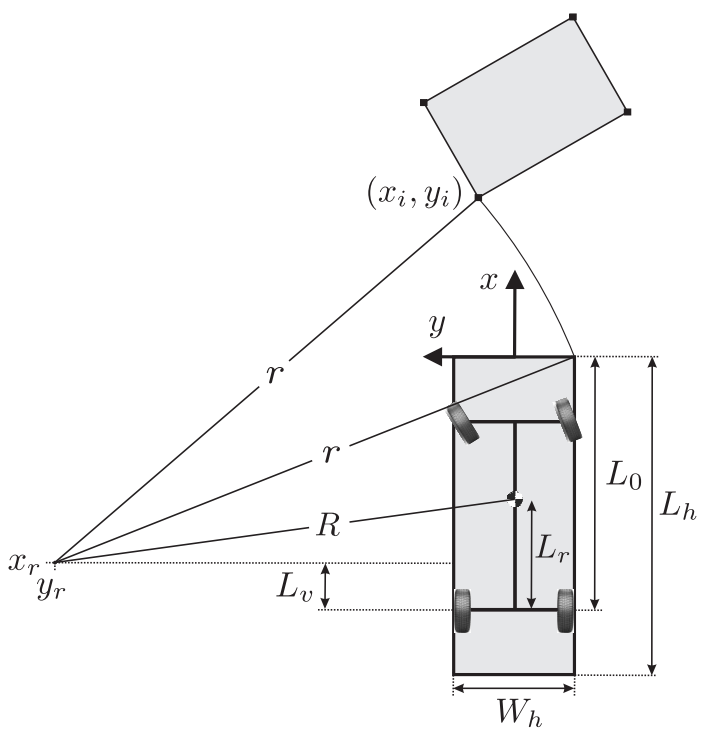

Fig. 3. The host vehicle position at time $t=0$ is illustrated along with the polygon representing the predicted position of the target vehicle at a discrete time $t_{i}$. The figure illustrates the steering manoeuvre that the driver of the host vehicle can use to pass a corner of the object, $\left(x_{i}, y_{i}\right)$, with the front end of the host vehicle, at a discrete time instance $t_{i}$.

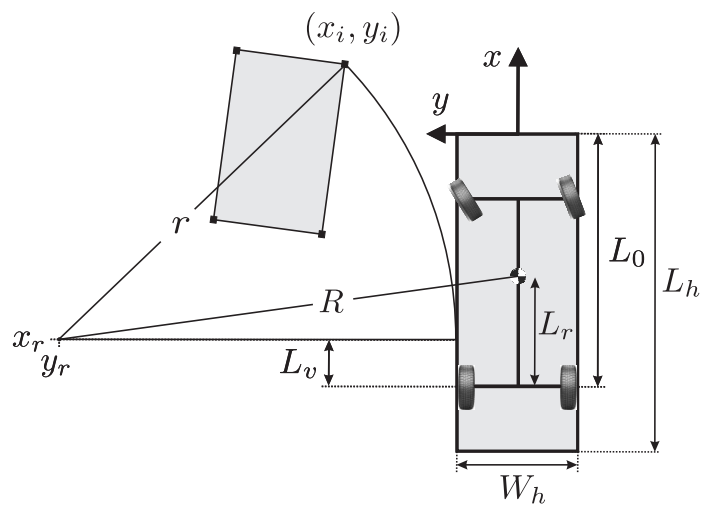

Fig. 4. The host vehicle position at time $t=0$ is illustrated along with the polygon representing the predicted position of the target vehicle at a discrete time $t_{i}$. The figure illustrates the steering manoeuvre that the driver of the host vehicle can use to pass a corner of the object, $\left(x_{i}, y_{i}\right)$, with the rear end of the host vehicle. Note that another time instance is illustrated in this figure than in Fig. 3. Compare with the illustration in Fig. 2.

$$
R_{\text {right }}=\operatorname{sign}\left(y_{r, \text { right }}\right) \sqrt{y_{r, \text { right }}^{2}+\left(L_{r}-L_{v}\right)^{2}}
$$

It is judged that the driver can steer to avoid a collision if

$$
a_{\text {lat }}^{\text {left }} \leq a_{\text {lat }}^{\text {max }} \text { OR } a_{\text {lat }}^{\text {right }} \geq-a_{\text {lat }}^{\text {max }}
$$

Furthermore, the steering angle, given by (1), shall not exceed the maximum steering angle of the vehicle, $|\delta| \leq \delta^{\max }$.

\section{Brake or accelerate to avoid}

In this section, a method is proposed for estimating how the driver of the host vehicle can use braking or acceleration to avoid a collision. While braking or accelerating to avoid a collision, it is assumed that the turn center, $\left(x_{r}, y_{r}\right)$, does not 
change. Similarly to the steer to avoid method in Section IV-B, the method consist of these steps:

1) Predict the motion of the target vehicle with an arbitrary motion model during a limited prediction horizon, $t \in$ $\left[0, T_{\max }\right]$.

2) Divide the prediction horizon into a series of discrete time steps $t_{i}=i t_{s}$, e.g. $t_{s}=0.05 s$ where $i \in 1,2, . ., N$ and $t_{s}=T_{\max } / N$.

3) Let the target be represented by a polygon of arbitrary shape and number of vertices. The shape of the polygon can be changed at each time step. Denote the endpoints of all edges of the polygon as $\left(\mathbf{x}_{1, i}, \mathbf{y}_{1, i}\right)$ and $\left(\mathbf{x}_{2, i}, \mathbf{y}_{2, i}\right)$.

4) For each time step, $t_{i}$, find all edges of the polygon that the host vehicle has to avoid. Denote the endpoints of these edges with $\left(\mathbf{x}_{1, i}^{\prime}, \mathbf{y}_{1, i}^{\prime}\right)$ and $\left(\mathbf{x}_{2, i}^{\prime}, \mathbf{y}_{2, i}^{\prime}\right)$.

5) For each time step, $t_{i}$, estimate how acceleration or braking can be used to avoid a collision with all edges selected in step 4.

6) The union of the solutions in step 5 is used to estimate how the driver can brake or accelerate to avoid all edges during the entire prediction horizon.

Step 1-3 are straightforward and needs no further explanation. Step 4: Only edges that potentially can be reached by braking or acceleration has to be considered. Reachable edges fulfill

$$
\min \left(\mathbf{y}_{1, i}, \mathbf{y}_{2, i}\right) \leq \frac{W_{h}}{2} \text { AND } \max \left(\mathbf{y}_{1, i}, \mathbf{y}_{2, i}\right) \geq-\frac{W_{h}}{2}
$$

and are denoted as $\left[\left(\mathbf{x}_{1, i}^{\prime}, \mathbf{y}_{1, i}^{\prime}\right),\left(\mathbf{x}_{2, i}^{\prime}, \mathbf{y}_{2, i}^{\prime}\right)\right]$.

Step 5: Denote a single edge at time $t_{i}$ as $\left[\left(x_{1}, y_{1}\right),\left(x_{2}, y_{2}\right)\right]$, where $\left[\left(x_{1}, y_{1}\right),\left(x_{2}, y_{2}\right)\right] \in\left[\left(\mathbf{x}_{1, i}^{\prime}, \mathbf{y}_{1, i}^{\prime}\right),\left(\mathbf{x}_{2, i}^{\prime}, \mathbf{y}_{2, i}^{\prime}\right)\right]$. To avoid a collision with the edge, the host vehicle has to avoid the $x$ position interval $\left[x_{i}^{-}, x_{i}^{+}+L_{h}\right]$ at the time $t_{i}$, as illustrated in Fig. 5. The interval is given by

$$
\begin{aligned}
& x_{i}^{-}=x_{1}+\min \left(\left(x_{2}-x_{1}\right) \frac{y^{\text {left }}-y_{1}}{y_{2}-y_{1}},\left(x_{2}-x_{1}\right) \frac{y^{\text {right }}-y_{1}}{y_{2}-y_{1}}\right) \\
& x_{i}^{+}=x_{1}+\max \left(\left(x_{2}-x_{1}\right) \frac{y^{\text {left }}-y_{1}}{y_{2}-y_{1}},\left(x_{2}-x_{1}\right) \frac{y^{\text {right }}-y_{1}}{y_{2}-y_{1}}\right)
\end{aligned}
$$

where

$$
\begin{aligned}
y^{\text {left }} & =\min \left(\frac{W_{h}}{2}, \max \left(y_{1}, y_{2}\right)\right) \\
y^{\text {right }} & =\max \left(-\frac{W_{h}}{2}, \min \left(y_{1}, y_{2}\right)\right)
\end{aligned}
$$

In order to avoid collisions where the host vehicle comes to a rest and then is hit from the side or front, let

$$
x_{i}^{-}=\min \left(x_{i}^{-}, x_{i+1}^{-}\right) \forall i \in 1,2, \ldots, N-1
$$

If $\min \left(x_{i}^{-}\right) \leq 0$, a collision can not be avoided by braking.

Let the host vehicle acceleration be described by an acceleration profile with one degree of freedom, e.g.

$$
a(t)= \begin{cases}a_{0}+j_{r} t & \text { if } t \in\left[0, t_{j}\right] \\ a_{r} & \text { if } t>t_{j}\end{cases}
$$

as illustrated in Fig.6, where $a_{0}$ is the initial host vehicle acceleration and $a_{r}=a_{0}+j_{r} t_{j}$ the final acceleration. The

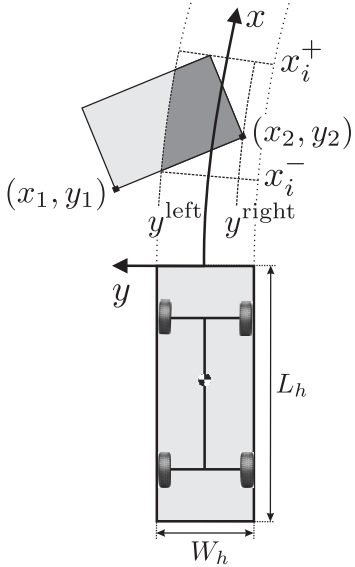

Fig. 5. The host vehicle is illustrated as time $t=0$ and the the predicted position of the target vehicle at time $t_{i}$. The polygonal shape of the target is divided into edges, where every edge has to be avoided. One edge, $\left[\left(x_{1}, y_{1}\right),\left(x_{2}, y_{2}\right)\right]$, is illustrated in the figure along with $y^{\text {left }}$ and $y^{\text {right }}$ for the edge and the distances $x_{i}^{-}$and $x_{i}^{-}$for the entire polygon.

acceleration rate $j_{r}$ is a variable which gives the acceleration profile one degree of freedom. A suitable selection of $t_{j}$ is

$$
t_{j}=\max \left(0, \frac{a_{\min }-a_{0}}{j_{\min }}\right)
$$

where $a_{\min }$ is the maximum deceleration of the host vehicle and $j_{\min }$ is the maximum deceleration rate.

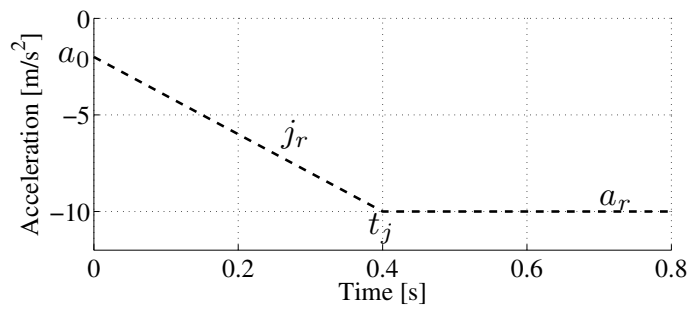

Fig. 6. Example of an acceleration profile (22), where the initial acceleration, $a_{0}=-2 \mathrm{~m} / \mathrm{s}^{2}, t_{j}=0.4 \mathrm{~s}, a_{r}=-10 \mathrm{~m} / \mathrm{s}^{2}$ and $j_{r}=-20 \mathrm{~m} / \mathrm{s}^{3}$.

Since the acceleration profile only has one degree of freedom, there is only one way to travel a certain distance in a given time. The derivation of the variable $j_{r}$ for traveling a distance, $x_{i}$, in a given time, $t_{i}$, is given by

$$
j_{r}=\frac{x_{i}-v_{0} \tilde{t_{j}}-a_{0} \frac{\tilde{t}_{j}^{2}}{2}-\left(t_{i}-\tilde{t_{j}}\right)\left(v+a_{0} \tilde{t_{j}}\right)-a_{0} \frac{\left(t_{i}-\tilde{t_{j}}\right)^{2}}{2}}{\frac{\tilde{t}_{j}^{3}}{6}+\left(t_{i}-\tilde{t_{j}}\right) \frac{\tilde{t}_{j}^{2}}{2}+\tilde{t_{j}} \frac{\left(t_{i}-\tilde{t_{j}}\right)^{2}}{2}}
$$

where $\tilde{t_{j}}=\min \left(t_{j}, t_{i}\right)$. To avoid the edge, $\left[\left(x_{1}, y_{1}\right),\left(x_{2}, y_{2}\right)\right]$, by accelerating, put $x_{i}=x_{i}^{+}+L_{h}$ in (24) and denote the solution as $j_{r}^{+}$. The needed acceleration is then given by $a_{r}^{+}=a_{0}+j_{r}^{+} t_{j}$. To avoid the edge by braking, put $x_{i}=x_{i}^{-}$in (24) and denote the solution as $j_{r}^{-}$. The needed deceleration is then given by $a_{r}^{-}=a_{0}+j_{r}^{-} t_{j}$. Store the solutions for all time steps and all selected edges in two vectors, $\mathbf{a}_{r}^{+}$and $\mathbf{a}_{r}^{-}$. 
Step 6: To avoid all edges during the entire prediction horizon, the driver of the host vehicle must decelerate at least $a_{\text {brake }}=\min \left(\mathbf{a}_{r}^{-}\right)$or accelerate at least $a_{\text {accelerate }}=\max \left(\mathbf{a}_{r}^{+}\right)$. It is judged that the driver can avoid a collision by braking if $a_{\text {brake }} \geq a_{\min }$ and by accelerating if $a_{\text {accelerate }} \leq a_{\max }$, where $a_{\max }$ is the maximum acceleration of the host vehicle, e.g. $a_{\max }=4 \mathrm{~m} / \mathrm{s}^{2}$.

\section{RESULTS FROM SIMULATIONS}

Let a collision avoidance system (CA) be based on the decision-making algorithm in Section III and the method for threat assessment in Section IV. The CA system has been evaluated on the traffic scenarios described below.

Let a target vehicle slow down to $5 \mathrm{~km} / \mathrm{h}$ before initiating a $90^{\circ}$ turn with a $12 \mathrm{~m}$ radius of turn. The target is accelerating with $1 \mathrm{~m} / \mathrm{s}^{2}$ while turning. The host vehicle drives straight through the intersection at constant speed. The host vehicle speed and initial position are varied to create different scenarios. For both vehicles, assume that $a_{\mathrm{lat}}^{\max }=8 \mathrm{~m} / \mathrm{s}^{2}, \delta^{\max }=45^{\circ}$, $a_{\max }=4 \mathrm{~m} / \mathrm{s}^{2}, a_{\min }=-10 \mathrm{~m} / \mathrm{s}^{2}, j_{\min }=-20 \mathrm{~m} / \mathrm{s}^{2}, t_{d}=0.3 \mathrm{~s}$, $W=2 \mathrm{~m}, L=5 \mathrm{~m}, L_{f}=1.12 \mathrm{~m}, L_{r}=1.68 \mathrm{~m}$ and $k=0.01$, as described in Section IV. Denote the time to collision as $t_{c}$.

The simulations show that autonomous braking is initiated up to $1 \mathrm{~s}$ before a potential collision. The autonomous braking reduces the impact velocity significantly, especially when the initial host vehicle velocity is $v \in[40,60] \mathrm{km} / \mathrm{h}$. Impact velocity reductions of up to $40 \mathrm{~km} / \mathrm{h}$ are observed. Some collisions are avoided for host vehicle velocities up to $30 \mathrm{~km} / \mathrm{h}$.

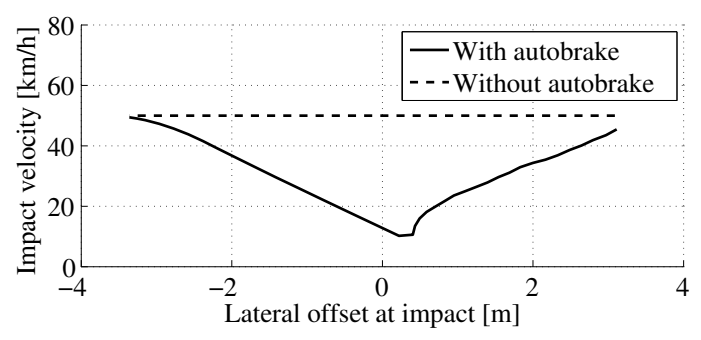

Fig. 7. Example of simulations with and without the CA system.

\section{CONCLUSIONS}

In this paper, a novel method has been presented for estimating how the driver of a vehicle can manoeuvre to avoid a collision with a moving object. In the method, it is assumed that the motion of the object can be described with an arbitrary motion model and that polygons can be used to describe its extension. The key idea in the method is to estimate how a collision can be avoided at discrete times. The solutions for all times are joined to obtain an estimate how to avoid a collision during the complete prediction horizon. This problem can be solved analytically for each time instance, under the assumption that the motion of the vehicle has one degree of freedom for steering and one for accelerating or braking. As an example, an analytical solution has been presented for a bicycle model where vehicle slip and brake dynamics are taken

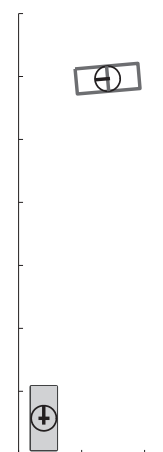

(a) $t_{\mathrm{c}}=1.5 \mathrm{~s}$

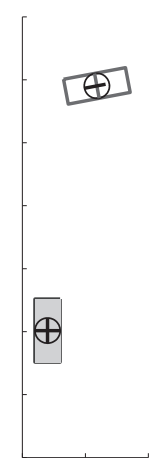

(b) $t_{\mathrm{c}}=1.0 \mathrm{~s}$

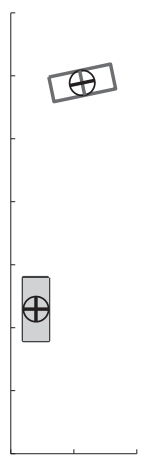

(c) $t_{\mathrm{c}}=0.9 \mathrm{~s}$ (d) $t_{\mathrm{c}}=0.0 \mathrm{~s}$

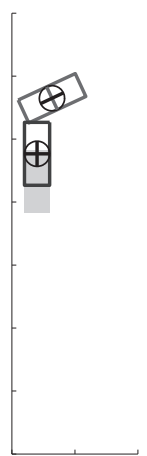

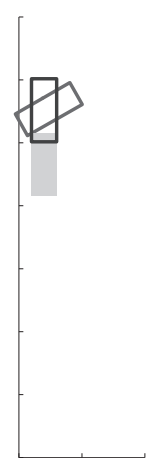

(e) $t_{\mathrm{c}}=-0.3 \mathrm{~s}$
Fig. 8. The host vehicle (bottom) is approaching the target vehicle (top) at $50 \mathrm{~km} / \mathrm{h}$. The gray vehicle is equipped with a CA system. The cross on top of the vehicles indicates how the driver of the vehicle can steer, brake or accelerate to avoid a collision. In a), it is easy for the target to avoid a collision by braking. In b), it is impossible for the host vehicle to avoid a collision by braking, steering or accelerating, while the target vehicle still can avoid a collision. In c), autonomous braking is initiated in the host vehicle to mitigate the collision. In d), the host vehicle without the CA system collides. In e), the host vehicle with a CA system collides. The duration of the brake intervention is $1.2 \mathrm{~s}$ and the impact velocity is reduced with $34 \mathrm{~km} / \mathrm{h}$. Note that a CA system in the target vehicle could avoid the collision by braking in b).

into account when judging if the driver of the vehicle can avoid a collision.

A collision avoidance by braking system, based on the proposed method and a new decision-making algorithm for autonomous braking, has been evaluated on simulated traffic scenarios. The simulations indicate that there is a high potential for using autonomous braking to avoid or mitigate collisions at intersections.

\section{ACKNOWLEDGMENT}

The authors would like to thank the Intelligent Vehicle Safety Systems (IVSS) programme for sponsoring this work.

\section{REFERENCES}

[1] M. Peden, R. Scurfield, D. Sleet, D. Mohan, A. Hyder, E. Jarawan, C. Mathers, World report on road traffic injury prevention, World Health Organization, The World Bank, Geneva, 2004.

[2] V.L. Neale, T.A. Dingus, S.G. Klauer, J. Sudweeks and M. Goodman, An overview of the 100 car naturalistic study and findings, ESV, 2005.

[3] J. Jansson, Collision Avoidance Theory with Application to Automotive Collision Mitigation Dissertation No. 950, Sweden, 2005.

[4] R.R. Knipling, J.S. Wang and H.M. Yin, Rear-End Crashes: Problem Size Assessment and Statistical Description, Report No: DOT HS 807 994, Washington, DC: NHTSA, 1993.

[5] E. Coelingh, H. Lind, W. Birk and D. Wetterberg, Collision Warning with Auto Brake, FISITA World Congress, Yokohama Japan, 2006.

[6] Traffic safety facts 2006 - A Compilation of Motor Vehicle Crash Data from the Fatality Analysis Reporting System and the General Estimates System, Report No: DOT HS 810 818, NHTSA, 2006.

[7] W.G. Najm, J.D. Smith, D.L. Smith, Analysis of crossing path crashes, Report No: DOT HS 809 423, NHTSA, 2001.

[8] J. Hillenbrand, A.M. Spieker and K. Kroschel, A Multilevel Collision Mitigation Approach - Its Situation Assessment, Decision Making and Performance Tradeoffs, IEEE Transactions on Intelligent Transportation Systems, Vol. 7, No. 4, pages 528-540, IEEE, 2006.

[9] A. Eidehall and L. Petersson, Threat assessment of general road scenes using Monte Carlo sampling, Proceedings of the IEEE Intelligent Transportation Systems, pages 1173-1178, 2006.

[10] T.D. Gillespie, Fundamentals of Vehicle Dynamics, Society of Automotive Engineers, SAE R-114, 1992, ISBN 1-56091-199-9 